\title{
Acquired von Willebrand syndrome associated with indolent systemic mastocytosis
}

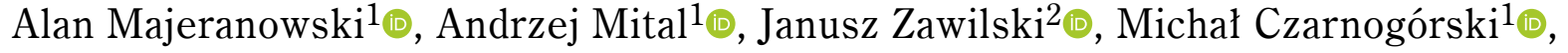 \\ Aleksandra Janowiak-Majeranowska ${ }^{3}$ (1) \\ ${ }^{1}$ Department of Hematology and Transplantology, Medical University of Gdansk \\ ${ }^{2}$ Diagnostic and Therapeutic Center InterLab in Poznan \\ ${ }^{3}$ Department of Otolaryngology, Medical University of Gdansk
}

\begin{abstract}
Hereby we present case report of acquired von Willebrand syndrome (AVWS) in patient with indolent systemic mastocytosis (ISM). Systemic mastocytosis may be considered as a model disease for development of AVWS. Thereinafter, pathophysiology of the disease as well as diagnostic and therapeutic approach were extensively discussed.
\end{abstract}

\section{Key words: acquired von Willebrand syndrome, systemic mastocytosis, hemorrhagic diathesis}

J. Transf. Med. 2020; 13: 151-152

\section{Introduction}

Acquired von Willebrand syndrome is a hemorrhagic diathesis, with clinical symptoms similar to those associated with the inherited von Willebrand disease. AVWS is characterized by lack of previous bleeding symptoms, negative family history, occurrence in older age. It develops in the course of other conditions, for example: cardiovascular, proliferative or autoimmune disorders, furthermore, it can be associated with some non-hematological malignancies and use of certain drugs. Pathogenesis of von Willebrand syndrome is complex. Deficiency or impaired activity of von Willebrand factor can result from the presence of specific antibodies against this factor, its adsorption onto the surfaces of neoplastic cells, mechanic damage or proteolysis.

Mastocytosis is a neoplastic condition characterized by pathological accumulation, proliferation and infiltration of mast cells into the tissues of the body. Mast cells have tendency to degranulate and release specific mediators and cytokines such as interleukins, proteoglycans, histamine and proteases, that induce clinical symptoms of disease [1-3].

\section{Case report}

A 45-year-old woman was diagnosed in 2009 with indolent systemic mastocytosis, that met all 3 minor criteria: KIT D816V mutation, KIT-positive, CD25 + mast cells present and elevated level of serum tryptase $(20-25 \mathrm{ng} / \mathrm{ml})$, but not the major criterion: multifocal dense infiltration contained only 10 mast cells in one infiltration (with criterion $>15$ ). Presented clinical symptoms were both cutaneous and systemic: urticaria pigmentosa, flushing, abdominal discomfort, bone pain and fatigue. Patient was treated with standard doses of ibandronic acid, alendronic acid, calcium carbonate, alfacalcidolum, fexofenadine, montelukast and ranitidinum with positive clinical and biochemical effect - tryptase level decreased below $20 \mathrm{ng} / \mathrm{ml}$.

Correspondence address: lek. Alan Majeranowski, Department of Hematology and Transplantology, Medical University of Gdansk, Debinki 7 street, 80-952 Gdansk, Poland, e-mail: a.majeranowski@gumed.edu.pl

Translation: lek. Alan Majeranowski 
During a follow-up visit in 2012 patient reported symptoms of haemorrhagic diathesis epistaxis, menorrhagia and purpura, which started initially in 2011. The family history of bleeding disorders was negative.

The laboratory tests performed in 2012 revealed a decreased level of fibrinogen $-1,41 \mathrm{~g} / \mathrm{L}$ $(1,80-3,50 \mathrm{~g} / \mathrm{L})$ and factor VII - 49\% (50-200\%), as a result of which, patient was diagnosed with hypofibrinogenemia and factor VII deficiency.

From 2012 to 2014 ISM was well-controlled with tryptase level below $20 \mathrm{ng} / \mathrm{ml}$. The most important symptoms that remained were epistaxis and menorrhagia. In 2014 medical team decided to extend diagnostics targeting particularly at haemorrhagic disorders. The laboratory analysis discovered decreased levels of: concentration of vWF (vWF: Ag) - 45\% (50-160\%); the activity of $\mathrm{vWF}$ as ristocetin cofactor (vWF:RCo) $-43 \%$ (60-170\%) and ability to bind to collagen (vWF: CB) $-47 \%$ (55-140\%). According to this, another diagnosis was established - acquired von Willebrand syndrome.

\section{Discussion}

In conclusion, systemic mastocytosis can be analyzed as model disease for the development of acquired von Willebrand syndrome. A decreased concentration of $\mathrm{vWF}$ can be explained by:

- loss of high-molecular-weight multimers of vWF resulting from their exposure to the proteolytic enzymes released during mast cells degranulation: tryptase, chymase and carboxypeptidase A3;

- adsorption of vWF on neoplastic mast cells leading to its enhanced clearance [4].

Due to that, more than one of the mechanisms mentioned above could be involved. This implies, that patients with mastocytosis require extended diagnostics targeted at Acquired von Willebrand syndrome.
AVWS should be always considered in patients with novel bleeding whenever laboratory tests suggest von Willebrand Disease, especially in the presence of AVWS-associated disorders. AVWS testing is also recommended before major surgery or an intervention with a high risk of bleeding in any patient with an AVWS-associated disorder. Treatment of the underlying condition using immunosuppressants, surgery, or chemotherapy, can lead to remission of AVWS in some individuals and should always be taken into consideration. Strategies to prevent and treat bleeding episodes should be focused on the use of VWF-containing factor VIII concentrates, desmopressin and tranexamic acid, but mostly, treatment success will depend on the underlying pathogenesis of the disease [4-7].

\section{References}

1. Metcalfe DD. Mast cells and mastocytosis. Blood. 2008; 112(4): 946-956, doi: 10.1182/blood-2007-11-078097, indexed in Pubmed: 18684881.

2. Dahlin JS, Hallgren J. Mast cell progenitors: origin, development and migration to tissues. Mol Immunol. 2015; 63(1): 9-17, doi: 10.1016/j.molimm.2014.01.018, indexed in Pubmed: 24598075.

3. Federici A. Acquired von Willebrand syndrome: an underdiagnosed and misdiagnosed bleeding complication in patients with lymphoproliferative and myeloproliferative disorders. Semin Hematol. 2006; 43: S48-S58, doi: 10.1053/j.seminhematol.2005.11.003.

4. Mital A. Acquired von Willebrand Syndrome. Adv Clin Exp Med. 2016; 25 (6): 1337-1344.

5. Collins P, Budde U, Rand JH, et al. Epidemiology and general guidelines of the management of acquired haemophilia and von Willebrand syndrome. Haemophilia. 2008; 14 Suppl 3: 49-55, doi: 10.1111/j.1365-2516.2008.01745.x, indexed in Pubmed: 18510522.

6. Tiede A. Diagnosis and treatment of acquired von Willebrand syndrome. Thrombosis Research. 2012; 130: S2-S6, doi: 10.1016/s0049-3848(13)70003-3.

7. Mital A, Prejzner W, Hellmann A. Acquired von Willebrand syndrome during systemic mastocytosis: an analysis of 21 cases. Pol Arch Intern Med. 2018; 128(7-8): 491-493, doi: 10.20452/ pamw.4295, indexed in Pubmed: 30057374. 